\title{
Impf-Alltag im 19. Jahrhundert
}

Das Verhältnis zwischen Ärzten und Bevölkerung vor dem Hintergrund der Pockenschutzimpfung im Kanton Luzern ${ }^{1}$

Mathias Steinmann

\section{Summary}

This study describes the vaccination practices in a rural area in Switzerland in the 19th century. Using reports of the vaccinating physicians and records of the local authorities a differentiated picture of the interests and behaviors of the involved social groups was obtained. Efforts at compulsory vaccination were counteracted by a variety of factors like shortage of vaccine, opposition by the people on the grounds of interference with private lives and mistrust in modern medicine and academically trained physicians, and even reservation by the vaccinating physicians themselves. Nevertheless, the practice of vaccination became a significant factor in the general process of medicalization, thus improving the position of the medical profession and furthering the public health movement by strengthening the bonds between medical knowledge and state power.

\section{Zusammenfassung}

Die folgende Untersuchung thematisiert den Impfalltag in einer ländlichen Gegend im 19. Jahrhundert. Aus den Rechenschaftsberichten der Impfärzte, aus Berichten der Gemeindebehörden und der Geistlichkeit liess sich ein sehr dichtes Bild der Interessen und Verhaltensweisen der beteiligten Personengruppen rekonstruieren. Die Bemühungen zur Durchsetzung der Impfpflicht

1 Der vorliegende Aufsatz basiert auf meiner Lizentiatsarbeit «Einführung und Verbreitung der Vakzination im Kanton Luzern», eingereicht am Historischen Seminar der Universität Basel 1993.

Mathias Steinmann, Historiker, Neustadtstrasse 4, CH-6003 Luzern 
wurden einerseits durch organisatorische Schwierigkeiten (Mangel an Impfstoff) erschwert, vor allem aber durch Widerstände in der Bevölkerung gegenüber obrigkeitlicher Eingriffe in die Privatsphäre sowie dem Misstrauen gegenüber der modernen Medizin und akademisch gebildeten Ärzten. Die Interpretation dieser Impfpraxis zeigt nun auch die Bedeutung der Vakzination im Prozess der Medikalisierung. Sie trug zu einem Statusgewinn und zur Professionalisierung der Ärzteschaft bei, dehnte den «Gesundheitsmarkt» auf die ganze Gesellschaft aus, veränderte das Arzt-Patienten-Verhältnis und verstärkte die Verbindung von staatlicher Macht und ärztlichem Wissen.

\section{Die gesetzliche Impfpflicht}

Bereits 1804, nur einige Jahre nachdem der englische Landarzt Edward Jenner die Entdeckung der Schutzkraft der Lymphe der Kuhpocken gegen die schwarzen Blattern veröffentlichte, ${ }^{2}$ propagierten einige Luzerner Ärzte im Kantonsblatt die Einführung und Verbreitung der Kuhpocken «als Schutzmittel gegen die Menschenblattern». ${ }^{3}$ Wer sich gegen die Impfung stelle, wende sich nicht nur gegen die Vernunft und mache sich gegenüber seinen Mitmenschen schuldig, sondern handle letztlich auch gegen die göttliche Vorsehung, so die Argumentation der anonymen Autoren. Doch erst 1819 erliess die Regierung eine Impfordnung, mit der vorerst alle medizinischen Laien vom Impfgeschäft ausgeschlossen wurden. Einige Jahre später wurden die Waisenkinder der direkten Impfpflicht unterstellt. Dieser erste Schritt zur Einführung des Impfzwanges erfolgte mit der Absicht, mit einem zentral organisierten Ablauf des Impfgeschäftes und der Impfpflicht einer bestimmten Anzahl Kinder die Versorgung des Zentraldepots mit frischem Impfstoff jederzeit zu gewährleisten. Die Impfung der Waisenkinder wurde nach Bezirken gestaffelt vorgenommen, und die Ärzte wurden verpflichtet, jeweils frische, von gesunden Kindern entnommene Lymphe dem Sanitätsrat zu senden. In der Folge einer grösseren Pockenepidemie in den Jahren 1826/27 erliess die Regierung die Verordnung, als Bedingung für die Schulaufnahme sei der Nachweis der Impfung vorzulegen. Auf ein allgemeines Impfobligatorium wurde angesichts des Widerstandes der Landbevölkerung vorerst verzichtet, obwohl die Ärzteschaft die zwangsweise Durchimpfung der ganzen Bevölkerung mit Vehemenz forderte. Die Behörden richteten ihre medi-

2 E. Jenner, An inquiry into the causes and effects of Variola Vaccinae. A disease, discovered in some of the western counties of England, particularly Gloucestershire, and known by the name of Cow-Pox, London 1798.

3 Luzernisches Kantonsblatt Nr. 4ff., 1804. 
zinalpolitischen Strategien vielmehr nach den politischen und gesellschaftlichen Möglichkeiten. Der Impfzwang wurde von der konservativen Restaurationsregierung vor allem deshalb abgelehnt, weil sie die fehlende Akzeptanz der Vakzination und der gesundheitspolizeilichen Massnahmen als politisches Risiko einschätzte. Erst die neue liberale Regierung führte unter der Leitung des Arztes und Präsidenten des Santitätskollegiums Jakob Robert Steiger 1834 das Impfobligatorium ein.

In den folgenden Jahrzehnten lässt sich im Impfwesen eine relative Kontinuität feststellen. Die gesetzlich vorgeschriebene Impfpflicht wurde zwar vollzogen, doch ohne besonderen Eifer, weder seitens der Behörden noch der Ärzte. Viele Eltern widersetzten sich dem Zwang und liessen ihre Kinder ungeimpft, ohne Sanktionen zu befürchten, trotz den angedrohten Strafverfolgungen. Die Zahl der jährlichen Impfungen schwankte denn auch sehr stark, nur während den Epidemienjahren waren die Vakzinationen jeweils besonders hoch. Der Widerstand gegen die staatliche Impfpolitik war in dieser Zeit somit vor allem passiver und individueller Art.

In der zweiten Hälfte des Jahrhunderts begann nun aber auch eine öffentliche Auseinandersetzung und Diskussion um die Impfung und insbesondere um den Impfzwang. Ärztliche und politische Impfkritiker lehnten den Impfzwang aus medizinischen und rechtlichen Gründen ab.

In der Folge der Pockenepidemie 1879/80 und im Vorfeld der eidgenössischen Abstimmung über ein Seuchengesetz 1882 entbrannte auch in Luzern eine heftige Diskussion über den Sinn und die Zweckmässigkeit der gesetzlichen Impfpflicht. Im Januar 1882 beschlossen der National- und Ständerat mit grosser Mehrheit das Bundesgesetz betreffend Massnahmen gegen gemeingefährliche Epidemien. ${ }^{4}$ Die einzelnen Gesetzesbestimmungen sahen vor, die Bekämpfung ansteckender Krankheiten auf eidgenössischer Ebene zu reglementieren und waren mit Ausnahme des Impfwesens unbestritten. Im Artikel 13 wurde jedoch die gesetzliche Impfpflicht vorgeschrieben. «Jedes in der Schweiz geborene Kind soll in der Regel im ersten, spätestens im zweiten Lebensjahr geimpft werden.» Obwohl der gesetzliche Impfzwang in den meisten Kantonen bereits seit Jahren eingeführt war - nur in den Kantonen Uri, Glarus und Genf war die Impfung freigestellt - und der eidgenössische Gesetzestext weitgehend den kantonalen Impfordnungen entsprach, mobilisierte der Artikel 13 und vor allem auch die vorgesehenen Strafbestimmungen die Impfgegner. ${ }^{5}$ Innert Monatsfrist wurden über 80000

4 Bundesgesetz betreffend Massnahmen gegen gemeingefährliche Epidemien,31. Januar 1882; in Schweizerisches Bundesblatt 1882, Nr. 7.

5 ebd. § 21. Bei Nichtbeachtung oder Umgehung der Vorschriften konnte eine Busse von bis zu Fr. 1000.- oder eine Gefängnisstrafe bis auf sechs Monate ausgesprochen werden. Im Wiederholungsfall konnte das Strafmass auf das Doppelte erhöht werden. 
Unterschriften für ein Referendum gesammelt. Am 30. Juli 1882 kam es zur Volksabstimmung. Das Bundesgesetz wurde mit einer grossen Mehrheit von 253968 Nein gegen 67820 Ja verworfen. Alle Kantone bis auf Neuenburg lehnten das Epidemiengesetz ebenfalls deutlich ab, im Kanton Luzern stimmten 10481 gegen und bloss 1779 für die Vorlage. Auch wenn die massive Ablehnung im Kontext der Referendumsstürme gesehen werden muss - der Versuch, der auf Bundesebene politisch einflusslosen katholisch-konservativen Partei mittels Referendum auf das Bundesgeschehen Einfluss zu nehmen und mit föderalistischen Argumenten gegen jegliche eidgenössischen Gesetze zu kämpfen -, zeigt sich hier auch die weitverbreitete Abneigung und das Unbehagen weiter Teile der Bevölkerung gegen die Impfung. Krankheiten erschienen oft noch als ein natürlicher und gottgewollter Gang der Dinge, während die Vakzination fremder Stoffe einen nicht legitimen Eingriff in die natürliche Ordnung bedeutete. Das klare Abstimmungsresultat zeigt aber auch ein geringes Vertrauen der Bevölkerung in die ärztlichfachliche Autorität. Die Ärzteschaft hatte sich in ihrer grossen Mehrheit für die Beibehaltung des Impfzwanges ausgesprochen.

\section{Der Impf-Alltag}

«Wie ich einem früheren Schreiben mitgeteilt, steht es mit der Impfung für einen Arzt, der seine Pflicht erfüllen möchte, wie ich bis anhin, so verdriesslich, dass man allen Muth verlieren müsste. Von Oben her Order, von Unten her Renitenz. In der Mitte steth der Impfarzt mit seinem Verdruss. $»^{6}$

Mit diesen Worten schilderte ein Impfarzt dem Sanitätsrat des Kantons Luzern im Jahre 1880 seine Erfahrungen mit der gesetzlich verordneten Pockenschutzimpfung. Wie der Bericht zeigt, war das Impfgeschäft für viele Ärzte eine verdriessliche Angelegenheit, die sie oft nur widerwillig ausübten. Vergegenwärtigt man sich die Organisation und den Ablauf der Impfung, wird dieser ärztliche Missmut deutlicher und konkreter.

Nachdem der Dorfpfarrer oder der Gemeindeammann als Vollzugsbeamter der Regierung den Impftermin in der Gemeinde bekanntgegeben hatte, kam der Impfarzt (vom Sanitätsrat bestimmte, zum Impfen verpflichtete Ärzte) ins Dorf, um an einigen Kindern die Vorimpfung vorzunehmen. Einige Tage später entnahm er den Pusteln dieser Kinder die humanisierte Lymphe und führte die allgemeine Impfung durch. Schliesslich musste der Impfarzt ein letztes Mal in die betreffenden Gemeinden reisen, um den Erfolg der Impfung zu kontrollieren. Insgesamt waren so mindestens zwei oder

6 Staatsarchiv Luzern (StALU) 34/64 B, Impfarzt Fischer an den Sanitätsrat, 4. Juni 1880. 
drei Besuche in einem Impfkreis zu machen, und die Impfärzte hatten meist mehrere Impfkreise zu betreuen. Dieser Ablauf war aus ärztlicher Sicht jedoch mit zahlreichen Hindernissen und Mühseligkeiten verbunden.

«Der Impfarzt opfert einen ganzen Tag Zeit, begibt sich auf dem Lande in eine Entfernung von einigen Stunden und zwar nach gesetzlicher Vorschrift zweymal, nämlich zur Impfung und zur Besichtigung, dafür ist ihm die Taxe von 5 Batzen zugesichert, da nach seinem Bescheinen 4-6 Kinder zum impfen sich einfinden, oft auch gar keine.» ${ }^{7}$

Mehr als 50 Jahre später äusserte ein Impfarzt den gleichen Missmut:

«Es werden wohl bald die Ärzte gezählt sein, die aus reinem Patriotismus eine Wegstrecke von bis zu 8 Kilometern bei gutem oder schlechtem Wetter der Impfresultate zuliebe machen werden.» ${ }^{8}$

Die Wegstrecken zu den oft entfernt gelegenen Dörfern waren weit und beschwerlich, vielfach blieb der Reiseaufwand der Impfärzte erfolglos, die impfpflichtigen Kinder erschienen nicht, oder nur einige wenige, die Kinder waren plötzlich alle krank, und manchmal war die Vorimpfung erfolglos: der Impfstoff wirkte nicht, die Kinder kratzten ihre Pusteln auf, so dass keine humanisierte Lymphe vorhanden war, die Entnahme der Lymphe in die Gläschen war oft schwierig, weil die Kinder zappelten, schrien und sich wehrten. Ein Impfarzt berichtete dem Sanitätsrat:

«Als ich das erste Mal zum Zweck des Vorimpfens mich nach dort begab, waren die hierzu tauglichen Kinder am Keuchhusten krank, beim zweiten Besuch war die Impflymphe nichts Nutz und beim dritten Besuch wollte die Mutter nicht mehr impfen lassen.»"

Um den Eltern die Vorimpfung beliebter zu machen, war es den Impfärzten seit 1871 erlaubt, Eltern, die ganz gesunde Kinder zum Vorimpfen und zur Stoffabnahme gaben, eine Gratifikation von 1-2 Franken zu bezahlen. ${ }^{10}$ Mit dieser Methode schien die Obrigkeit die Eltern zu ködern, doch die willkürliche Praxis der Verteilung dieser Gratifikation führte gleichzeitig zu neuen Konflikten. Die regierungsrätliche Interpretation dieser Verordnung sah zwar «eine Berechtigung des Impfarztes zur Erteilung einer Gratifikation, nicht aber die Verpflichtung vor». ${ }^{11}$ So erklärte Impfarzt Grüter, die Gratifikation werde nur an «arme und weitherkommende Mütter» ausbezahlt, «der Neid und die Missgunst aber von vielen wurde dadurch sehr rege gemacht.» ${ }^{12}$

7 StALU BG 100/1, Bemerkungen des Impfarztes von Mettenwil 1824.

8 StALU 34/64 B, Impfarzt Dubach an den Sanitätsrat, 9. Okt. 1881.

9 ebd.

10 Verordnung der Santitätskommission, 2. März 1871.

11 StALU 34/65, Militär- und Polizeidep. an den Sanitätsrat, 20. Sept. 1882.

12 StALU 34/36 B, Impfarzt Grüter an den Sanitätsrat, 22. Okt. 1877. Einer Lehrersfamilie wurde die Gratifikation verweigert, weil von den zwei vorgeimpften Kindern nur bei einem Lymphe entnommen werden konnte. Der Lehrer beschwerte sich in der Folge bei der Sanitätsbehörde: «Ersuche Sie letzteres (Auszahlung der Gratifikation) bis längstens den 14. Juli 1877 zu thun, ansonsten ich mich sofort an ein Departement wenden werde. Dann ver- 
Der Ärger und Verdruss der Impfärzte beruhte nicht nur auf diesen äusseren Umständen im Ablauf des Impfgeschäftes, sondern richtete sich auch gegen die Sanitätsbehörde und die Organisation des Impfwesens. Der Impfstoffmangel und die unterschiedliche Qualität der Lymphe waren die zentralen Probleme des Impfwesens. Die Klagen über die Qualität der Impfmaterie tauchten in den meisten Arztberichten immer wieder auf.

«Es zeigte sich, dass der mir gegebene Impfstoff die erwartete Wirkung nicht hervorbrachte, so weil er wahrscheinlich auch gealtert war; und es war daher zu befürchten, wenn man damit zu impfen fortführe, es möchte die natürlichen Pocken nachher bey diesen Kindern ausbrechen, und das wohlthätige Impfgeschäft der Schutzpocken-Impfung bey dem nicht vaccinierten Volke in einen bösen Ruf bringen.» ${ }^{13}$

Ein anderer Arzt berichtete über Nebenwirkungen der Impfung:

«Allein die bedenklichen Erscheinungen die sich gleich anfangs zu den von verschiedenen Stoffen vaccinierten Kindern gesellte: die häufig angeschwollenen axillar Drüsen, das zu früh zerplatzenden Nässen der Blatter, die verschiedenartigen Ausschläge, die häufige und bedenkliche Kränklichkeit der Kinder, welche mit diesen erhaltenen Stoffen geimpft wurden, bestimmten mich, diesen ganzen Stoff eingehen zu lassen.» ${ }^{14}$

Die Sanitätsbehörde war kaum je in der Lage, die Impfärzte mit genügend Impfstoff zu versorgen sowie die Qualität dieser Lymphe zu garantieren. Die Einrichtung eines zentralen Impfdepots und die Gewinnung von animaler Lymphe erwiesen sich als untaugliche Versuche, diesen Mangel zu beheben.

Die Sanitätsbehörde zeigte aber auch in der Vollziehung der Impfordnung wenig Sensibilität und Durchsetzungsvermögen. Die Strafbestimmungen gegenüber renitenten Eltern wurden nie vollzogen und erwiesen sich als «leere Drohungen». Die Ärzteschaft verlangte von der Regierung denn auch wiederholt die Verschärfung der Kontrolle und die konsequente Vollziehung der Impfordnung mit den gesetzlich vorgesehenen Strafbestimmungen. ${ }^{15}$ War die Obrigkeit gegen widersetzliche Eltern ziemlich nachsichtig, erwies sie sich aber gegenüber den Ärzten oft unflexibel. Die Regierung erachtete den Einsatz der Ärzte im Impfwesen und auch im Armenarztwesen als selbstverständlichen ärztlichen Dienst «für die öffentliche Gesundheit», da die Ärzte ihrerseits auch staatliche Protektion genössen. ${ }^{16}$ Ärzte, die gegen die Anweisungen der Sanitätsbehörde opponierten, wurden mit der Drohung des Patent-Entzuges zurechtgewiesen. Vielfach wurden die zahlreichen Ideen und Anregungen der Ärzteschaft von der Sanitätsbehörde ignoriert. Neben

lange ich keine Gratifikation, sondern eine Entschädigung, umso eher, da das Kind, wie es sehr deutlich zeigt, heftig angegriffen ist.»

13 StALU 24/36 C, Bezirksarzt Krauer an die Sanitätskommission, 3. April 1824.

14 StALU 24/36 C, Bezirksarzt Scherrer an die Sanitätskommission, 29. April 1824.

15 StALU 34/64 B, Referat von Dr. Jenni über die Impfung im Jahre 1881, siehe auch StALU 34/36 B, Referat von Dr. Röösli, Sektion Sursee der ärztlichen Gesellschaft, 28. Dez. 1876. 16 StALU 34/36 B, Auszug aus dem Verhandlungsprotokoll des Regierungsrates, 4. Juli 1881. 
Verbesserungsvorschlägen, wie die ausschliessliche Verwendung von animaler Lymphe oder der Anstellung eines staatlichen Impfarztes mit fixer Besoldung, verlangten die Ärzte immer wieder die Erhöhung ihres Honorars. Sie glaubten, dass bei einer besseren Bezahlung die Ärzte das Impfen viel engagierter betreiben und vor allem die unbezahlte Kontrolle über den Erfolg der Impfung gewissenhafter durchführen würden. An der Jahresversammlung des kantonalen ärztlichen Vereins 1872 wurde die fehlende $\mathrm{Zu}$ sammenarbeit zwischen den Impfärzten und der Sanitätsbehörde kritisiert.

«Es muss die Überzeugung zum Durchbruch gelangen, dass das Medizinalpersonal und die Sanitätsbehörde nicht im Antagonismus stehen, sondern Grössen oder Mächte, oder wie sie es nennen wollen, sind, die nach den selben Zielen zu streben haben, nach Hebung des Gesundheitswesens. ${ }^{17}$

Fasst man all diese grossen und kleinen Schwierigkeiten im Impf-Alltag zusammen, wird deutlich, wie sehr die Verbreitung und die Akzeptanz der Vakzination auch von der Organisation des Impfwesens abhing. Vor allem die Ärzte, die als Mediziner die Impfung befürworteten, sahen sich in ihren Bemühungen um die Bekämpung der Pocken durch diese Probleme stark behindert. Doch auch die Sanitätsbehörde musste selbstkritisch feststellen, «wie leidig alle willkürlichen Abweichungen endlich auf den Erfolg und die Wirksamkeit der besten Absichten wirken, sie sehen, wie alles dadurch gelähmt und wirkungslos in ein Chaos sich auflöst.» ${ }^{18}$ Vergegenwärtigt man sich zudem, dass die Ärzte mit der Impflanzette im Kontakt mit der Bevölkerung meist auf Abneigung und Misstrauen stiessen, wird nur allzudeutlich, dass das Impfgeschäft für viele Ärzte ein «verdriessliches» war.

Aus der Lektüre der zahlreichen Arztberichte während der Untersuchungsperiode (1804-1890) erscheint die «Renitenz von Unten» auf den ersten Blick als Widerstand von abergläubischen, vorurteilsvollen, störrischen und ungebildeten Personen, denen weder die Gesundheit ihrer eigenen Kinder noch das Gemeinwohl am Herzen liegt. Versucht man hingegen die Wahrnehmung des Impf-Alltags aus der Perspektive der betroffenen Eltern zu rekonstruieren, äussert sich diese Renitenz viel differenzierter. Man muss sich die Situation erneut vergegenwärtigen: Nachdem der Pfarrer oder der Gemeindeammann den Impftermin in der Gemeinde bekanntgegeben hatte, kam der Arzt ins Dorf, um die Impfung vorzunehmen. Vielleicht war dieser Arzt aus seiner Erfahrung mit der Impfung bereits schlecht gelaunt, nach der längeren Reise in der heissen Nachmittagssonne oder im Regen erwartete ihn noch mehr Verdruss; nur wenige, aber schreiende und zappelnde Impf- 
linge, eine misstrauische Bevölkerung, «störrische Mütter», kurz eine «üble Stimmung, die bei den meisten Einwohnern gegen das Impfen herrschte». ${ }^{19}$

Diese üble Stimmung gegen die Impfung hatte die verschiedensten Gründe. Man kann von einem grossen sozialen und «kulturellen Graben» ${ }^{20}$ zwischen der Ärzteschaft und der Landbevölkerung ausgehen, der die Verständigung stark erschwerte. Auf der einen Seite der akademisch gebildete, fortschrittsorientierte Arzt und auf der anderen Seite die einfache, traditionalistische Landbevölkerung. Diese Gegenüberstellung ist zwar stark vereinfacht und schematisiert, doch die verschiedenen, oft beiläufigen Bemerkungen in den Arztberichten verdeutlichen eine aus dieser Kluft herrührende Spannung zwischen den beiden sozialen Gruppen. Einerseits bemühten sich die Ärzte zwar um einen möglichst guten und konfliktfreien Umgang mit der Bevölkerung. So berichtete ein Arzt über seine Erfahrungen mit den Müttern der zu impfenden Kinder:

«Ich muss vielen Müttern, deren Kinder ich Lymphe enthebe, immer einige Batzen Schmerzensgeld blechen und dabei noch sehr höflich und ordelig mit den Leuten reden.» ${ }^{21}$

Anderseits zeigt die Betonung dieses Umstandes, dass es wahrscheinlich oft nicht der Fall war, dass man «höflich und ordelig» miteinander sprach. Die Arztberichte vermitteln im Gegenteil viele Vorurteile und eine oft geringschätzende Meinung der Ärzte über die Landbevölkerung. ${ }^{22}$ Viele Menschen standen aber auch der Arbeit des Arztes feindselig gegenüber. «Dem einen war der Arzt zu jung, dem anderen zu kokett, einem dritten zu habgierig.» ${ }^{23}$ Sehr anschaulich beschrieb auch Jeremias Gotthelf dieses Misstrauen gegen den Arzt. Als Anne Bäbi Jowäger einen Arzt holen liess, sprach ihr Mann:

«Er wolle aber Freude haben, wenn alles gut komme, man könne dann sehen, was so ein Doktor abtrage, ob er für etwas anderes da sei, als den Leuten das Geld abzstehle und den lieben Gott taub z'machen mit seiner Hochmut.» ${ }^{24}$

19 StALU 24/36 C, Bericht des Impfarztes Geisseler über das Impfen im Impfkreis Wohlhusen, 5. August 1831.

20 A. Corbin, Kulissen. In: Geschichte des privaten Lebens. Von der Revolution zum Grossen Krieg. Hg. von M. Perrot, Bd. 4. Frankfurt a.M. 1992, 613.

21 StALU 34/64 A, Impfarzt Suppiger an die Santitätskommission 10. Juni 1876.

22 «Vorurtheile und Dummheit sind noch an vielen Orten zu finden» Impfarzt Suppiger 10. Juni 1876, StALU 34/64 A. «Will man ein solcher Trotzkopf zur Verantwortung ziehen, so kann ich im voraus versichern, dass seine allfällige Entschuldigung lauter Lügen sind» StALU BG 100/2. «Widersetzlichkeit mancher Eltern und zum Theil auch in den Wundertreibern, Quacksalbern und alten Weibern ausgeheckten und in dieser, in ihrer geistigen Entwicklung vielleicht um ein halbes Jahrhundert zurückgebliebenen Gemeinde umhergebotenen Vorurtheile, als seyen die Menschenpocken eine Strafe oder wenigstens ein Verhängnis Gottes, welcher der Mensch nur mit der Gefahr eine Sünde zu begehen, entgegen wirken dürfe.» StALU BG 100/7.

23 A. Corbin, Kulissen. Frankfurt a.M. 1992, 613.

24 J. Gotthelf, Wie Anne Bäbi Jowäger haushaltet und wie es ihm mit dem Doktern geht. Solothurn 1843,37 . 
Dieser Gegensatz manifestierte sich vor allem dann, wenn konkrete Konflikte ausbrachen. So beschwerte sich ein Familienvater, dessen Ehefrau keine Gratifikation ausbezahlt erhielt, bei der Regierung über den Impfarzt:

«Statt aber diesem vollberechtigten Verlangen zu entsprechen, fand der Impfarzt für gut den Unterzeichneten mit groben Worten abzuschnauzen. $»^{25}$

Dieser Gegensatz wurde akzentuiert durch die Tatsache, dass die meisten Ärzte in ihren Impfkreisen ortsfremd und erst nach ihrer Ausbildung in diese Gegend gezogen waren. Die Sanitätsbehörden und die Ärzteschaft äusserten denn auch immer wieder den Wunsch, es mögen die Ärzte in ihnen bekannten und vertrauten Gemeinden impfen. Der Dorf- oder Hausarzt sei als Impfarzt vorzuziehen, denn dieser sei mit den Familienverhältnissen, auch aus medizinischer Sicht, besser vertraut. ${ }^{26}$ Angesichts der geringen Ärztedichte auf dem Land war diese Idee nicht realisierbar. Die Impfordnungen erlaubten zwar seit 1828 grundsätzlich die freie Wahl des Impfarztes.

«Wer für sich oder seine Kinder aus besonderem Zutrauen einen anderen, als den zur Impfung aufgestellten Arzt, zu gebrauchen wünscht, dem stehet es frey.» ${ }^{27}$

Da die Kosten der Privatimpfung jedoch selbst bezahlt werden mussten, ist anzunehmen, dass diese Möglichkeit nur selten genutzt wurde.

Ein weiterer Grund für die «üble Stimmung» war die Vakzination selbst. Ohne Zweifel war die Renitenz gegen das Impfen auch ein Widerstand gegen obrigkeitliche Eingriffe in die Privat- und Intimsphäre, aber auch Aberglauben und 'fortschrittsfeindliche Ablehnung' der Moderne. Viele Einwände gegen die Impfung waren jedoch konkret medizinisch begründet: das mangelhafte Wissen über das Wesen der Pocken und über die Verbreitung der Infektion, die Unsicherheit über den Sinn und Nutzen der Vakzination, die Ängste aufgrund der unterschiedlichen Qualität des Impfstoffes. Meldungen und Gerüchte über Impfschäden verbreiteten sich denn auch sehr schnell in der Öffentlichkeit. Allein das Prinzip der Impfung, dass in Zeiten keiner gesundheitlicher Gefährdung und ohne die Symptome einer Erkrankung trotzdem eine medizinische Versorgung angestrebt und betrieben

25 StALU 34/65 Beschwerde von Schreiner Meier an den Regierungsrat 24. Juli 1882.

26 StALU 24/36 C, Bezirksarzt Rüttimann an die Sanitätskommission, 19. Juni 1821. Vgl. auch StALU 34/36 B, Impfarzt Gilli an den Sanitätsrat 21. Jänner 1889. «Es ist ein Unsinn von solchen Kindern Impfstoff zu nehmen, deren Eltern man in sanitarischer Hinsicht nicht kenne.» In Preussen wurde aus ähnlichen Überlegungen gegen den Widerstand der akademischen Ärzte den Wundärzten, Pfarrern, Lehrern und Hebammen die Impfung erlaubt. U. Frevert, Krankheit als politisches Problem 1770-1880. Soziale Unterschichten in Preussen zwischen medizinischer Polizei und staatlicher Sozialversicherung. Göttingen 1984, 72.

27 Beschluss, einen geregelten Gang in der Impfung der Schutzpocken anordnend, §15 (Sammlung der Gesetze und Regierungsverordnungen für die Stadt und Republik Luzern 1814-1831). 
wurde, war neu. Nahmen im Ancien Régime der Arzt und die Gesundheitspolizei während der Epidemien eine dominante Stellung ein, wurde mit der Idee der Prophylaxe (Pockenschutzimpfung) auch die seuchenfreie, die 'gesunde' Zeit von der medizinischen Versorgung erfasst. Schliesslich war auch die Idee, einen tierischen Krankheitsträger in einen gesunden, menschlichen Körper einzuimpfen, für Teile der Bevölkerung wahrscheinlich kaum nachvollziehbar. So wurde beispielsweise die gefährlichere Variolation, die Einimpfung von Menschenpocken, noch Jahrzehnte nach der Einführung der Vakzination verschiedentlich ausgeübt; in der Impfordnung von 1828 wurde diese Praxis denn auch explizit verboten. ${ }^{28}$

Das Problem der Impfstoffqualität wurde bereits erwähnt. Die Einimpfung eines fremden Krankheitskeimes, die Gefahr der Impfschäden und die Ungewissheit über die Schutzwirkung der Vakzination waren die realen Erfahrungen der Eltern mit den Kuhpocken. Besonders in den Anfängen der Impfung war es die Furcht vor

«bösen Folgen, als verschiedene Ausschlagskrankheiten, Schwerhörigkeit, böse Augen als Folge des früheren unkundigen Impfens, und des Impfens von ungesundem Stoff, die [...] dem guten Fortgang der Impfung viele Schwierigkeiten entgegensetzte.» ${ }^{29}$

Selbst die Sanitätskommission stellte 1829 angesichts der andauernden Widerspenstigkeit eine differenzierte Frage nach den Gründen dieser Renitenz und ordnete eine Untersuchung an,

«ob auch ein Verschulden des Impfarztes vorliegt oder ob nur die Vorurteile gegen das Impfen das Impfgeschäft dermassen behindere.» ${ }^{30}$

Es ist jedoch festzuhalten, dass trotz der weitverbreiteten Abneigung gegen das Impfen, trotz der mangelhaften Organisation des Impfwesens und der bisweilen geringen Begeisterung der Ärzte im Impf-Alltag, die Vakzination durchaus erfolgreich verbreitet wurde. Besonders während der Periode des Impfzwanges wurde ein grosser Teil der Neugeborenen geimpft. Die Mehrheit der Bevölkerung war somit geschützt gegen eine Pockeninfektion. Die Sanitätskommission bemerkte 1875 im Jahresbericht an das Sanitätskollegium:

«Was die Kinderimpfung anbelangt, so nehmen diesselben so ziemlich ihren regelmässigen Verlauf. Es treten zwar auch hier Schwankung im Impfeifer auf und steigt namentlich dieser Artikel ja mit Nähe der Gefahr und fällt mit dem Verschwinden derselben.» ${ }^{31}$

Bei den Eltern war, wie die Sanitätskommission feststellte, während der Epidemien das Einverständnis, ihre Kinder zu impfen, viel grösser als in den ru-

28 ebd. § 23, vgl. StALU 24/36 C, Bericht des Impfarztes Geisseler, 5. August 1831.

29 StALU 24/36 C, Bezirksarzt Rüttimann an die Sanitätskommission, 19. Juni 1821.

30 StALU 24/36 C.

31 StALU 34/64 A, 2. Feb. 1875. 


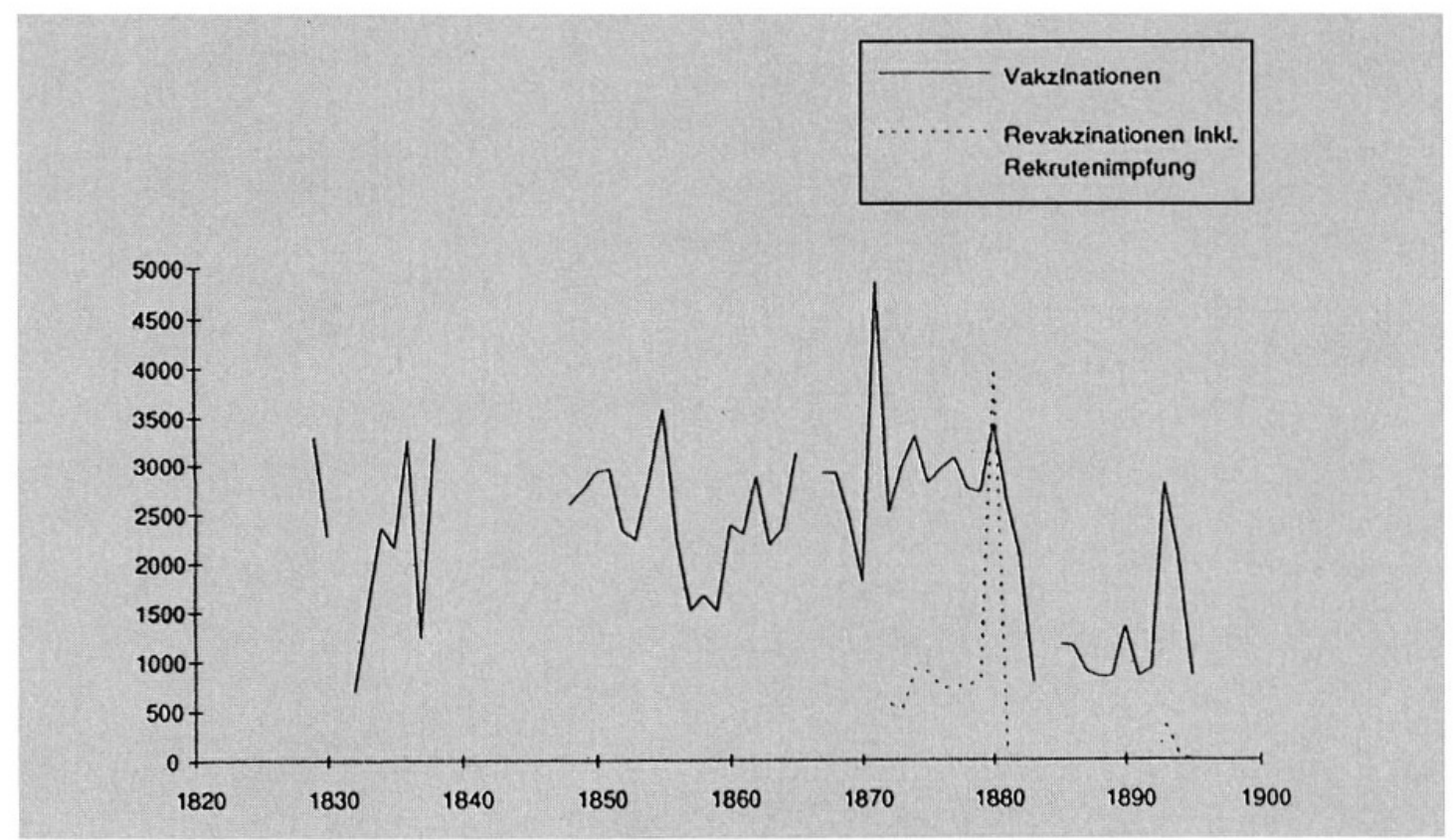

Fig. 1. Jährliche Impfungen im Kanton Luzern (1829-1895). Bemerkungen:

- Während der Epidemienjahre 1826-1830,1869-1871, 1879/80 und 1893/94 war die Zahl der jährlichen Impfungen besonders hoch.

- 1872-1881 Revakzinationspflicht für alle Rekruten.

- 1883 Aufhebung des kantonalen Impfzwanges.

- Fehlende Angaben: 1831,1839-1847 (Sonderbundsperiode), 1866, 1884.

higen Zeiten (vgl. Fig. 1). Aus verschiedenen Gemeinden wurde gar der ausdrückliche Wunsch der Eltern, ihre Kinder zu impfen, gemeldet. Diese positiven Resultate waren zweifellos auch ein Verdienst der Impfärzte, denn auch von ihrem Engagement hing es ab, wie gross die Bereitschaft der Bevölkerung zur Impfung war. Viele Ärzte waren bestrebt, ihre Pflicht als Impfärzte mit bestem Willen zu erfüllen. So schrieb Dr. Suppiger an die Sanitätskommission:

«Ich werde mir eine Ehre daraus machen, meine Mission möglichst gut zu erfüllen und zwar nicht 'pecuniae causa' [...] Ich sehe es aus Liebe zur Sache und Ihnen zu gefallen, denn ich weiss, dass ein Sanitätsaktuar der gewissenhaft seine Pflicht erfüllt, auch nicht immer auf Rosen gebettet ist.» ${ }^{32}$

All dem Verdruss zum Trotz nahmen die Impfärzte die oft weiten Wegstrecken zu ihren Impfkreisen auf sich, organisierten Fuhrwerke zum Transport von weit entfernt wohnenden Kindern, versuchten die Eltern vom Nutzen der Impfung zu überzeugen und zahlten fleissig Gratifikationen, um die Eltern mit Geld für die Vorimpfung zu gewinnen.

Die Haltung der Bevölkerung gegenüber der Vakzination, wie sie oben beschrieben wurde, scheint auf den ersten Blick widersprüchlich. Es ist einer32 StALU 34/64 A, Impfarzt Suppiger an die Sanitätskommission, 16. Juni 1876. 
seits ein Wunsch nach Schutz vor den Pocken und das heisst auch die Bereitschaft, sich oder seine Kinder impfen zu lassen, feststellbar, aber gleichzeitig auch starkes Misstrauen gegenüber der Impfung. Dieser Widerspruch zeigt jedoch, dass der Wandlungsprozess und die Veränderungen der medizinischen Vorstellung und Praxis im 19. Jahrhundert keinesfalls als kontinuierliche Entwicklung verstanden werden darf, sondern von Brüchen und Diskontinuitäten gezeichnet war.

\section{Das Verhältnis zwischen Impfarzt und Bevölkerung}

Wie oben erwähnt, war der Impf-Alltag durch den grossen sozialen und kulturellen Graben zwischen der Ärzteschaft und der Bevölkerung gezeichnet. Dieses bereits aufgrund der äusseren Umstände antagonistische Verhältnis wurde durch die unterschiedliche Interessenlage der beiden Gruppen zusätzlich verstärkt. Das eigentliche Ziel der medizinischen Wissenschaft, die Pocken zu bekämpfen und zu kontrollieren sowie die allgemeine Gesundheit zu verbessern, hatte im Impf-Alltag nur eine geringe Bedeutung. Der ImpfAlltag war vielmehr geprägt durch die konkreten und pragmatischen Interessen der Handlungsträger. Die Bevölkerungsgruppen, die der Impfung misstrauten und diese ablehnten, versuchten durch die Maschen der Impfordnung zu schlüpfen und sich der Impflanzette zu entziehen. Die Impfärzte ihrerseits erachteten die Vakzination zwar als sinnvolle und wirksame Prophylaxe gegen die Pocken, die Impfung selbst übten sie aufgrund der vielfältigen Hindernisse zum Teil nur widerwillig aus.

Das «verdriessliche» Impfgeschäft brachte den Ärzten aber auch einen bedeutenden Vorteil. Mit der Impfung wurde ein neuer Markt für ein medizinisches Angebot geschaffen. Allein die Idee der Pockenschutzimpfung, die Bekämpfung und die Ausrottung der Pocken mittels Durchimpfung der ganzen Bevölkerung, bedingte eine vollständige medizinische und gesundheitspolizeiliche Erfassung derselben. Neue, bisher von der ärztlichen Versorgung weitgehend ausgeschlossene, auf Selbstmedikation, Laien und Quacksalber angewiesene Bevölkerungsteile wurden so der ärztlichen Praxis zugeführt. Mit der Einführung der gesetzlichen Impfpflicht wurde diese Ausweitung des ärztlichen Handlungsraumes obrigkeitlich legitimiert, der neue Gesundheitsmarkt erhielt eine «staatliche Rückendeckung». ${ }^{33}$ Viele Leute kamen durch den Impfzwang erstmals mit einem Arzt in Kontakt. Die

33 S. Brändli, Die Retter der leidenden Menschheit. Sozialgeschichte der Chirurgen und Ärzte auf der Zürcher Landschaft 1700-1850. Zürich 1990, 324. U. Frevert, Krankheit. Göttingen 1984. 
geringe Ärztedichte auf der Landschaft lag nicht nur am mangelhaften Angebot an ärztlicher Dienstleistung, sondern war auch das Resultat der geringen Nachfrage nach medizinischer Versorgung. Die Landbevölkerung war kaum bereit, am wenigsten für Kinder, finanzielle Mittel für ärztliche Dienste zu mobilisieren. Mit der Impfpflicht waren die Menschen gezwungen, diese Dienstleistung in Anspruch zu nehmen.

Im Impfgeschäft fanden sich obrigkeitliche und ärztliche Interessen. Die Ärzte waren in der Perspektive der Regierung Vollzugspersonen für die Durchsetzung der staatlich verordneten Schaffung und Kontrolle der allgemeinen Volksgesundheit, anderseits garantierte der Staat in den Impfordnungen die Privilegien und den Status der Ärzte innerhalb des Medizinalpersonals im Rahmen dieser Ausweitung des Gesundheitsmarktes. Mit dem Impfgeschäft war somit auch die Professionalisierung und der Statusgewinn der Ärzteschaft verbunden. Die mit der Verbreitung der Vakzination geschaffene und staatlich legitimierte «Expansion des Marktes für gesundheitliche Dienstleistungen» ermöglichte den Ärzten, sich aus ihrer oft patronageartigen Abhängigkeit von ihren Patienten, der Oberschichtsklientel zu lösen. ${ }^{34}$ Die ärztliche Versorgung wurde zu einem Gut für die ganze Gesellschaft; mit der Institution des Amtsarztes wurde die ärztlich-medizinische Pflege auf die Unterschichten ausgedehnt. Innerhalb dieses Prozesses wurde der Arzt zum medizinischen Experten, dessen Autorität und Kompetenz die Selbstmedikation und die medizinischen Laien allmählich verdrängte.

Verschiedene Untersuchungen haben übereinstimmend ergeben, dass die Pockenschutzimpfung ein zentrales Element des Medikalisierungsprozesses des 19. Jahrhunderts sei, weil hier auf der Seite der Bevölkerung ein Perzeptionswechsel zugunsten der ärztlichen Kompetenz feststellbar sei. In ihrer Studie «Der Aufstieg der Ärzte im 19. Jh.» identifiziert Claudia Huerkamp Medikalisierung als einen mentalitätsmässigen Wandel, in dessen Verlauf es für den Alltagsmenschen zunehmend selbstverständlich wurde, im Krankheitsfall den kranken Körper ärztlicher Kontrolle zu unterstellen und sich nach der Anweisung des Experten 'Arzt' zur Wiederherstellung seiner Gesundheit zu richten. ${ }^{35}$ Huerkamp, aber auch Sebastian Brändli und Ute Frevert sehen nun in der Impfung einen wichtigen Teil dieses mentalitätsmässigen Wandels, da die medizinische Versorgung im Zuge der Verbreitung der Vakzination die Selbstmedikation und Quacksalber verdrängte und alle gesellschaftlichen Schichten umfasste, und so die Voraussetzung für diesen Wandel schuf. Durch diese monopolartige Stellung beanspruchte die Ärzteschaft einen beträchtlichen Teil der medizinischen Versorgung.

34 C. Huerkamp, Der Aufstieg der Ärzte im 19. Jh. Göttingen 1985, 137. 35 ebd. 139. 
Diese These kann aber vor dem Hintergrund des Impf-Alltags nicht vorbehaltlos übernommen werden. Zweifellos zählte die Verbreitung der Vakzination, die Expansion des Gesundheitsmarktes auf neue Bevölkerungsteile, zu den zentralen Bereichen der Medikalisierung, doch darf dieser Prozess nicht als lineare Entwicklung verstanden werden. Gerade der anhaltende Widerstand dieser Bevölkerungsgruppen verweist auf Diskontinuitäten innerhalb des Medikalisierungsprozesses. Mit der Expansion des Gesundheitsmarktes und der medizinalpolitischen Erfassung der ganzen Bevölkerung war nicht gleichzeitig immer auch ein Perzeptionswandel zugunsten der ärztlichen Autorität und der fachlichen Kompetenz der Medizin zu beobachten. Diese Ausweitung der ärztlichen Praxis war hauptsächlich das Resultat der Medizinalgesetzgebung und weniger des wachsenden medizinischen Wissens und der ärztlichen Autorität. Die Ablehnung des Epidemiengesetzes und in der Folge der markante Rückgang der jährlichen Impfungen nach Aufhebung der gesetzlichen Impfpflicht unterstreichen diese Hypothese. Der Widerstand gegen das Impfen zeigt im Gegenteil, dass die ärztliche Autorität und Kompetenz vielfach auch bezweifelt und abgelehnt wurde. Das Misstrauen und die Ablehnung dieser medizinischen Entdeckung gründete auf der realen Erfahrung im Umgang mit dem Impfstoff und dem Impfarzt. Eine Erfahrung, die aus den oben aufgezeigten Gründen oft in einem krassen Gegensatz zur ärztlichen Autorität und Kompetenz stand. Diese Konstanz des Widerstandes und des Misstrauens, trotz der massiven Propaganda und der gesetzlich vorgeschriebenen Impfung, lassen vermuten, dass zumindest im Bereich des Impfwesens das medizinische Wissen und die Autorität des Arztes in Teilen der Bevölkerung nur eine geringe Durchsetzungskraft hatte. Die deutliche Ablehnung des eidgenössischen Epidemiengesetzes 1882 zeigte schliesslich, auch wenn verschiedene Gründe für die Verwerfung verantwortlich waren, dass die Idee der Vakzination auch nach rund 80 Jahren seit der Einführung der Schutzimpfung und nach rund 50 Jahren Impfpflicht in der Bevölkerung kaum tief verankert war, und dass das Engagement und die Autorität der Ärzte für die Impfung im Abstimmungskampf keine Wirkung zeigte.

Mit der Ablehnung des Epidemiengesetzes mussten die Ärzte auf die «staatliche Rückendeckung» wieder verzichten und die beanspruchten Vollmachten wieder abgeben. Die Medikalisierung der Gesellschaft war jedoch bereits soweit fortgeschritten, dass die moderne Medizin auch ohne staatliche Legitimation wirken konnte. Zumindest die Expansion des Gesundheitsmarktes auf die ganze Gesellschaft kann u.a. auch als Resultat des Impfwesens im 19. Jahrhundert bezeichnet werden. Die Ärzteschaft sicherte sich zudem mit der Impfung die oberste Position innerhalb der Hierarchie des 
Gesundheitswesens und beanspruchte auch den grössten Teil der medizinischen Versorgung der Bevölkerung. Um seinen Status und die Akzeptanz seiner fachlichen Kompetenz musste der Arzt aber weiter kämpfen. Der Vormarsch der Ärzte an die Spitze der bürgerlich-akademischen Elite war genausowenig vollzogen wie die Ausweitung der medizinischen Wissenschaft auf die meisten menschlichen Lebensbereiche.

Die Spannungen zwischen der Ärzteschaft und der Bevölkerung verweisen zudem auf einen grundsätzlichen Paradigmawechsel im Arzt-PatientenVerhältnis. Dieses war traditionellerweise gekennzeichnet durch die patronageartige Abhängigkeit der Ärzte von ihrer Oberschichtsklientel. Mit dem Ausbau der medizinischen Versorgung begann sich dieses Verhältnis zu wandeln. Zwischen Arzt und Patient wurde im Laufe des 19. Jahrhunderts ein Autoritätsgefälle etabliert, welches an «oberster Stelle den unbedingten Anspruch auf Gehorsam des Patienten» postulierte. ${ }^{36}$ Die Festsetzung dieses Autoritätsgefälles war gekennzeichnet durch die völlige Akzeptanz dieses Anspruches durch die Patienten. Die Haltung des Patienten sollte gekennzeichnet sein durch Vertrauen in die fachliche Kompetenz des Mediziners, und aus dieser Vertrauenshaltung heraus sollte er sich der ärztlichen Anordnung freiwillig unterwerfen. ${ }^{37}$ Aufgrund der konkreten Situation im Luzerner Impfwesen scheint das Arzt-Patienten-Verhältnis mitten in diesem Wandlungsprozess. Zum einen wurde die ärztliche Fachkompetenz von weiten Teilen der Bevölkerung immer noch in Frage gestellt und nicht vorbehaltlos akzeptiert, zum anderen verlangten die Ärzte zunehmend Gehorsam gegenüber ihrem Wissen und ihrem Dienst. Der ärztliche Ruf nach strenger Vollziehung der Impfgesetze ist eine Form dieses Autoritätsanspruches.

Die Ausweitung der ärztlichen Handlungskompetenz in der Seuchenbekämpfung markierte zudem den Einbruch der «Öffentlichkeit ins Private». ${ }^{38}$ Die traditionelle Seuchenpolitik richtete den Blick hauptsächlich auf Stadt- und Landesgrenzen und auf die öffentlichen Anlagen wie Märkte oder Brunnen, sie machte jedoch halt vor den Türen der privaten Gemächer. Mit der Durchsetzung der Lehre des Kontagionismus rückte das einzelne Individuum als potentieller Ansteckungsherd in den Mittelpunkt. Die ärztlichen Aufgaben, das frühzeitige Erkennen der Krankheit, die Isolierung des Kranken, die Prävention und die Prophylaxe vermischten die Grenzen zwischen öffentlichem und privatem Raum. Die Suche nach dem Krankheitskeim verlagerte den ärztlichen Blick von der durch Miasmen verseuch-

36 C. Huerkamp, Ärzte und Patienten. In: Medizinische Deutungsmacht im sozialen Wandel. Hg. Von A. Labisch und R. Spree, Bonn 1989, 66.

37 ebd. 66.

38 A. Corbin, Kulissen. Frankfurt a.M. 1992, 618. 


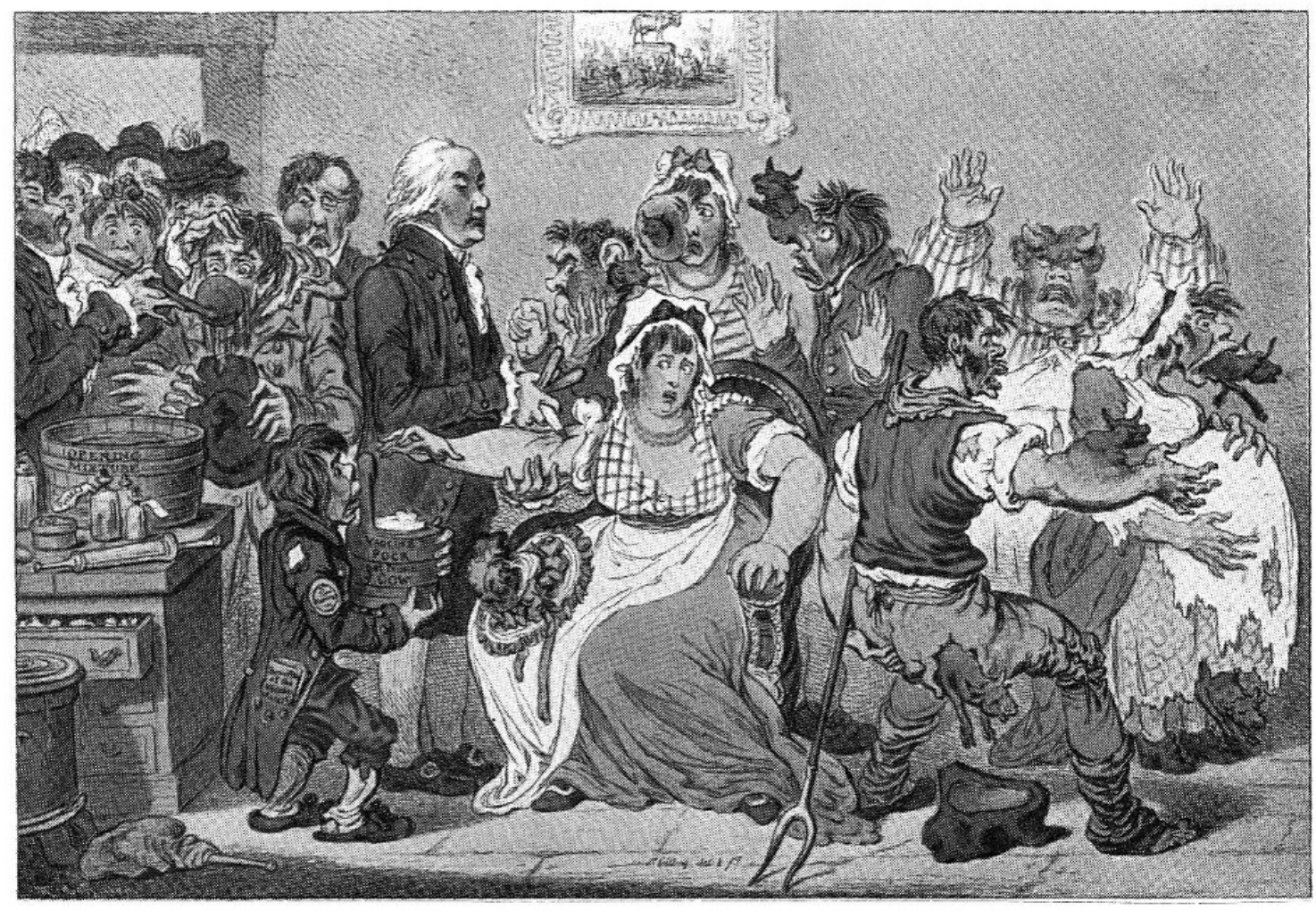

Abb. 1. J. Gillary, Dr. Jenner impft das britische Volk, sitzend Britannia, rechts hinten John Bull. Aus: Eugen Hollaender, Die Karikatur und Satire in der Medizin. Stuttgart 1905, 295.

ten Umwelt auch auf die sozialen Beziehungen. Die ärztliche Kompetenz reichte nun bis in die Gemächer der Menschen. ${ }^{39}$

Wie asymmetrisch das Arzt-Bevölkerung-Verhältnis war, zeigten die vielen Vorurteile der Ärzteschaft gegen die medizinischen Vorstellungen der Bevölkerung. Sie bezeichneten diese wie erwähnt als abergläubisch und vorurteilsvoll gegenüber dem medizinischen Wissen. Es gibt jedoch keine unmittelbaren Hinweise, wie stark ein solcher Aberglaube im Impfwesen tatsächlich verbreitet war. Die Karikatur darf nicht als Reflexion eines Aberglaubens verstanden werden. Die zahlreichen Impfkarikaturen müssen vielmehr als subversive Verspottung der medizinischen Wissenschaft und des ärztlichen Standes sowie auch als Propaganda in der Auseinandersetzung um den Impfzwang gesehen werden (vgl. Abb. 1). Die für die Vermittlung von schul- und volksmedizinischen Vorstellungen bedeutenden und populären Volkskalender zeigten ein etwas anderes Bild. In diesen Kalendern wurde

39 Die konkreten Auswirkungen der Pockenbekämpfung auf die soziale Hygiene waren sehr vielfältig und reichten von Desinfektionsvorschriften bis zur Körperpflege. So war der Impftermin beispielsweise zunehmend mit einer vorhergehenden Körperpflege verbunden. 
zur Blatternbekämpfung immer wieder die Schutzimpfung empfohlen. ${ }^{40}$ Die Unterschiede zwischen der Volksmedizin und der medizinischen Wissenschaft waren zum Teil geringer, als angenommen wurde. Ein charakteristisches Merkmal der Medizin der Aufklärung war die Übernahme und die Prüfung von Beobachtungen der Volksmedizin (z. B. Vakzination) $)^{41}$. Doch auch die Volksmedizin macht Anleihen bei den modernen Wissenschaften, ohne ihre innere Kohärenz aufzugeben. ${ }^{42}$ Dies lässt vermuten, dass das Prinzip und die Idee der Impfung der Bevölkerung durchaus vertraut war. Der vielzitierte Widerstand gegen das Impfen richtete sich eher gegen die Ausweitung der ärztlichen Handlungskompetenz und gegen den staatlichen Eingriff in die individuelle Gesundheitspflege und gründete auch auf der Skepsis gegenüber der medizinischen Ausführung der Impfung und der Qualität des Impfstoffes. Weite Teile der Bevölkerung vertrauten der Impfung, jedoch nicht im Sinne einer gesetzlich verordneten Prophylaxe, sondern eher im traditionalistischen Sinne einer gesundheitsfördernden Massnahme in Zeiten effektiver Seuchengefahr, wie die Zunahme der Vakzinationen während der Epidemien deutlich zeigte. Das Misstrauen gegen die Impfung war zum grossen Teil ein Misstrauen gegen die staatlich legitimierte und unterstützte Funktion der Impfärzte und die Ausweitung der ärztlichen Handlungskompetenzen.

40 B. C. Hansch-Mock, Deutschschweizerische Kalender des 19. Jahrhunderts als Vermittler schul- und volksmedizinischer Vorstellungen. Aarau 1976, 277.

41 E. H. Ackerknecht, Therapie von den Primitiven bis zum 20. Jh. Stuttgart 1970, 84. Zitiert nach J. P. Rupp, Die Entwicklung der Impfgesetzgebung in Hessen. In: Medizinhistorisches Journal Bd. 10 (1975) 104.

42 A. Corbin, Kulissen. Frankfurt a.M. 1992, 614. 